\title{
Blue lasing at room temperature in high quality factor GaN / AlInN microdisks with InGaN quantum wells
}

D. Simeonov, E. Feltin, H.-J. Bühlmann, T. Zhu, A. Castiglia, M. Mosca, J.-F. Carlin, R. Butté, and N. Grandjean

Citation: Appl. Phys. Lett. 90, 061106 (2007); doi: 10.1063/1.2460234

View online: https://doi.org/10.1063/1.2460234

View Table of Contents: http://aip.scitation.org/toc/apl/90/6

Published by the American Institute of Physics

\section{Articles you may be interested in}

III-Nitride-on-silicon microdisk lasers from the blue to the deep ultra-violet

Applied Physics Letters 109, 231101 (2016); 10.1063/1.4971357

Low threshold, room-temperature microdisk lasers in the blue spectral range

Applied Physics Letters 103, 021112 (2013); 10.1063/1.4813471

Lasing in GaN microdisks pivoted on $\mathrm{Si}$

Applied Physics Letters 89, 211101 (2006); 10.1063/1.2392673

Stimulated emission and lasing in whispering-gallery modes of GaN microdisk cavities

Applied Physics Letters 75, 166 (1999); 10.1063/1.124307

High quality nitride based microdisks obtained via selective wet etching of AllnN sacrificial layers

Applied Physics Letters 92, 171102 (2008); 10.1063/1.2917452

Optically pumped whispering-gallery mode lasing from $2-\mu \mathrm{m}$ GaN micro-disks pivoted on $\mathrm{Si}$

Applied Physics Letters 104, 221106 (2014); 10.1063/1.4881183

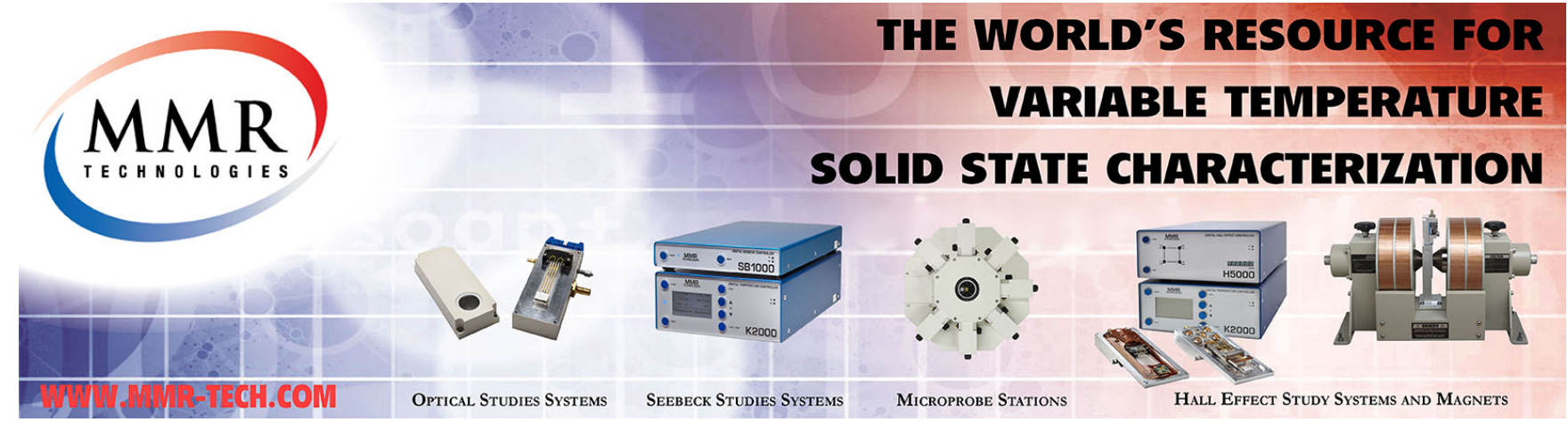




\title{
Blue lasing at room temperature in high quality factor GaN/AllnN microdisks with InGaN quantum wells
}

\author{
D. Simeonov, ${ }^{\text {a) }}$ E. Feltin, H.-J. Bühlmann, T. Zhu, A. Castiglia, ${ }^{\text {b) }}$ M. Mosca, ${ }^{\text {b) }}$ J.-F. Carlin, \\ R. Butté, and N. Grandjean \\ École Polytechnique Fédérale de Lausanne (EPFL), Institute of Quantum Electronics and Photonics, \\ CH-1015 Lausanne, Switzerland
}

(Received 27 November 2006; accepted 8 January 2007; published online 6 February 2007)

\begin{abstract}
The authors report on the achievement of optically pumped III-V nitride blue microdisk lasers operating at room temperature. Controlled wet chemical etching of an AlInN interlayer lattice matched to $\mathrm{GaN}$ allows forming inverted cone pedestals. Whispering gallery modes are observed in the photoluminescence spectra of $\mathrm{InGaN} / \mathrm{GaN}$ quantum wells embedded in the $\mathrm{GaN}$ microdisks. Typical quality factors of several thousands are found $(Q>4000)$. Laser action at $\sim 420 \mathrm{~nm}$ is achieved under pulsed excitation at room temperature for a peak power density of $400 \mathrm{~kW} / \mathrm{cm}^{2}$. The lasing emission linewidth is down to $0.033 \mathrm{~nm}$. (c) 2007 American Institute of Physics.
\end{abstract}

[DOI: 10.1063/1.2460234]

High quality factor $(Q=\lambda / \Delta \lambda)$ three-dimensional semiconductor microcavities (MCs) and microdisks ( $\mu$ disks) attract much attention because of their potential use for fabricating low-threshold lasers. ${ }^{1,2}$ They are equally interesting for studying cavity quantum electrodynamics (CQED) phenomena in the solid state such as strong light-matter coupling $^{3,4}$ or the Purcell effect. ${ }^{5,6}$ Furthermore embedding quantum dots (QDs) in such structures opens the way to the realization of single photon emitters. ${ }^{7}$ In this respect, III-V nitride semiconductors are particularly attractive for observing CQED phenomena at room temperature (RT) due to their large oscillator strength and exciton binding energies. ${ }^{8}$

Recent progress in the fabrication of planar nitride-based MCs has led to the successful demonstration of high $Q$ $(\sim 2800) \mathrm{MCs}^{9}{ }^{\text {as }}$ well as laser emission under optical pumping in vertical cavity surface emitting laser structures. ${ }^{10}$ The interest on semiconductor $\mu$ disks resides in their high $Q$ whispering gallery modes (WGMs) and their small modal volume. ${ }^{2}$ Furthermore, while the fabrication of high quality nitride distributed Bragg reflectors and MCs usually requires strain engineering solutions, ${ }^{11} \mu$ disks remain rather simple photonic structures in comparison. In addition, the latter possess the same advantages of compactness and ability to be integrated in two-dimensional arrays. However, the fabrication of $\mu$ disks has proven to be challenging in III-V nitrides mainly due to the lack of efficient underetching techniques. Indeed a convenient wet etching process suitable for the formation of thin disks $\left(d<\lambda / 2 n_{\text {eff }}\right)$ optically isolated from any surrounding higher index materials, as it is the case, e.g., for the III-V arsenide system, ${ }^{6}$ does not exist for standard AlGaN and InGaN alloys. Furthermore at short wavelengths, the emitted light is more sensitive to processing defects leading to scattering losses.

WGMs in nitrides were first observed in circular mesas. ${ }^{12}$ The mode structure was studied but neither lasing action nor high $Q$ factors were demonstrated. Recently the fabrication of nitride $\mu$ disks using photoelectrochemical band gap selective etching was successfully achieved leading

\footnotetext{
a)Electronic mail: dobri.simeonov@epfl.ch

b) Also at DIEET, Università degli Studi di Palermo, Palermo, Italy.
}

to disks optically isolated from the underneath template. ${ }^{13}$ Low temperature $(T=10 \mathrm{~K})$ lasing in these structures was reported under pulsed optical pumping with a peak power density threshold of $27 \mathrm{MW} / \mathrm{cm}^{2}$.

In this letter we report the fabrication of $\mu$ disk structures, containing InGaN quantum wells (QWs), in the latticematched GaN/AlInN system. Typical WGM $Q$ factors in the spontaneous emission regime of about 4000 are found. These $\mu$ disks exhibit a RT lasing in the blue wavelength range with a threshold at a peak power density of about $400 \mathrm{~kW} / \mathrm{cm}^{2}$.

The growth was carried out by metal organic vapor phase epitaxy (MOVPE) on 2 in. $c$-plane sapphire substrates. After deposition of a standard GaN template, a $200 \mathrm{~nm}$ thick $\mathrm{GaN}$ :Si doped layer is grown. It is followed by a $400 \mathrm{~nm}$ thick nonintentionally doped (nid) GaN layer to reduce the current flowing out of the mesas during the oxidation process. A $400 \mathrm{~nm}$ thick Si doped AlInN layer $\left(x_{\mathrm{In}} \sim 18 \%\right)$ nearly lattice matched to $\mathrm{GaN}$ is then deposited for subsequent lateral oxidation. The cavity and the active region are formed by a $100 \mathrm{~nm}$ thick $\mathrm{GaN}$ layer with three $\operatorname{In}_{0.15} \mathrm{Ga}_{0.85} \mathrm{~N}$ QWs about $3 \mathrm{~nm}$ thick separated by $12 \mathrm{~nm}$ thick $\mathrm{GaN}$ barriers.

After the MOVPE growth step, $200 \mathrm{~nm}$ thick circular $\mathrm{SiO}_{2}$ masks are formed and $550 \mathrm{~nm}$ high circular mesas $3-10 \mu \mathrm{m}$ in diameter are etched by $\mathrm{Cl}_{2} / \mathrm{Ar}$ reactive ion etching to give access to the GaN:nid layer and the AlInN layer sidewall. Then the bottom part of the AlInN layer is laterally etched using high current densities during a lateral oxidation process similar to that described in Ref. 14. This procedure gives access to the $\mathrm{N}$ polarity face of the AlInN layer. The mesas are then wet etched in a $\mathrm{HCl}: \mathrm{H}_{2} \mathrm{O}=1: 1$ solution. Here etching occurs both laterally and in the vertical direction through the previously revealed $\mathrm{N}$ polarity face. This process seems to be isotropic as no specific crystallographic planes appear (Fig. 1). After the etching step, the remaining protective $\mathrm{SiO}_{2}$ layer is dissolved in a $\mathrm{BHF}$ solution.

A typical $\mu$ disk structure is presented in the scanning electron microscope images in Fig. 1. The sidewall is smooth and inclined at $\sim 45^{\circ}$. In such $\mu$ disk structures, part of the emitted light is waveguided in the GaN layer. While the top 


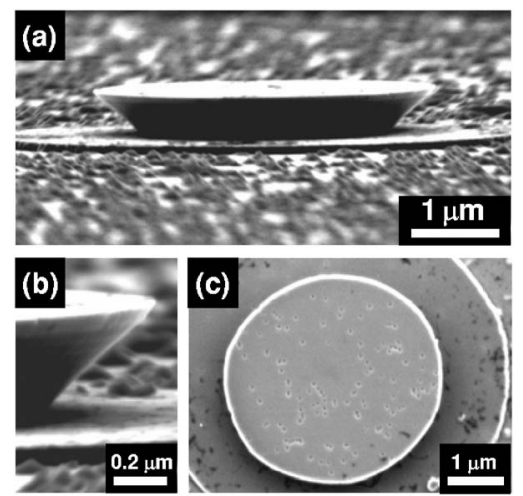

FIG. 1. Scanning electron micrograph of a $4 \mu \mathrm{m}$ diameter disk: (a) perspective view, (b) edge close-up view, and (c) top view.

confinement is only ensured by air, the bottom one is ensured by air at the edge of the disk and AlInN (refractive index $n \sim 2.28$ at $\lambda=420-440 \mathrm{~nm})^{15}$ in the central part. As a consequence, the effective refractive index $n_{\text {eff }}$ in the GaN waveguide varies between 2.10 and 2.28 when going from the edge to the central part of the disk. The guided light will be laterally confined by total internal reflections and thus will form a disk cavity.

The modal structure of the $\mu$ disks was investigated by photoluminescence (PL) using the $244 \mathrm{~nm}$ line of a cw $\mathrm{Ar}^{+}$ laser frequency doubling unit. Laser action was achieved using a pulsed $266 \mathrm{~nm}$ neodymium-doped yttrium aluminum garnet laser with an average output power of $2 \mathrm{~mW}$, a repetition rate of $8 \mathrm{kHz}$, and a pulse length of $500 \mathrm{ps}$. Measurements were performed in backscattering geometry using an UV microscope objective $(\times 80)$ with a numerical aperture of 0.55 . Samples were fixed normally to the optical axis. While recording spectra the laser is intentionally defocused in order to excite a whole $\mu$ disks ( $\sim 4 \mu \mathrm{m}$ in diameter). The spectra are recorded with a spectrometer offering a maximum spectral resolution of $0.019 \mathrm{~nm}$. All measurements were carried out at RT.

The $300 \mathrm{~K}$ PL spectrum of InGaN/GaN QWs before processing, measured under $\mathrm{cw}$ excitation, is presented in Fig. 2(a). The broad PL linewidth $(\sim 20 \mathrm{~nm})$ is a usual feature of InGaN QWs. No degradation of their optical properties due to the growth on AlInN instead of a GaN buffer is observed. Note that the multiple peak line shape originates from the interference occurring in the $\mathrm{GaN}$ template. For comparison, a PL spectrum of a single $\mu$ disk is presented in Fig. 2(b). It is worth emphasizing that such a broad luminescence allows probing simultaneously several optical modes, as it is the case with InAs QD ensemble (see below). ${ }^{16}$ The excitation power density of the cw laser was kept intentionally low, namely, $\sim 200 \mathrm{~W} / \mathrm{cm}^{2}$. Multiple modes are observed but narrow ones can be found only in the low energy part of the spectra. The modal picture suggests that they correspond to both TE and TM WGMs. ${ }^{12}$ The existence of higher order radial modes is also possible. However, their exact assignment requires complex simulations and will be the subject of future work. The narrowest linewidths are of the order of $0.07 \mathrm{~nm}$ but they are likely reduced by the short range interference due to internal reflections occurring in the sapphire substrate. After fitting the envelope of these oscillations we obtain typical linewidths of $\sim 0.1 \mathrm{~nm}$ corresponding to $Q$ factors of $\sim 4000$. These relatively high $Q$ factors

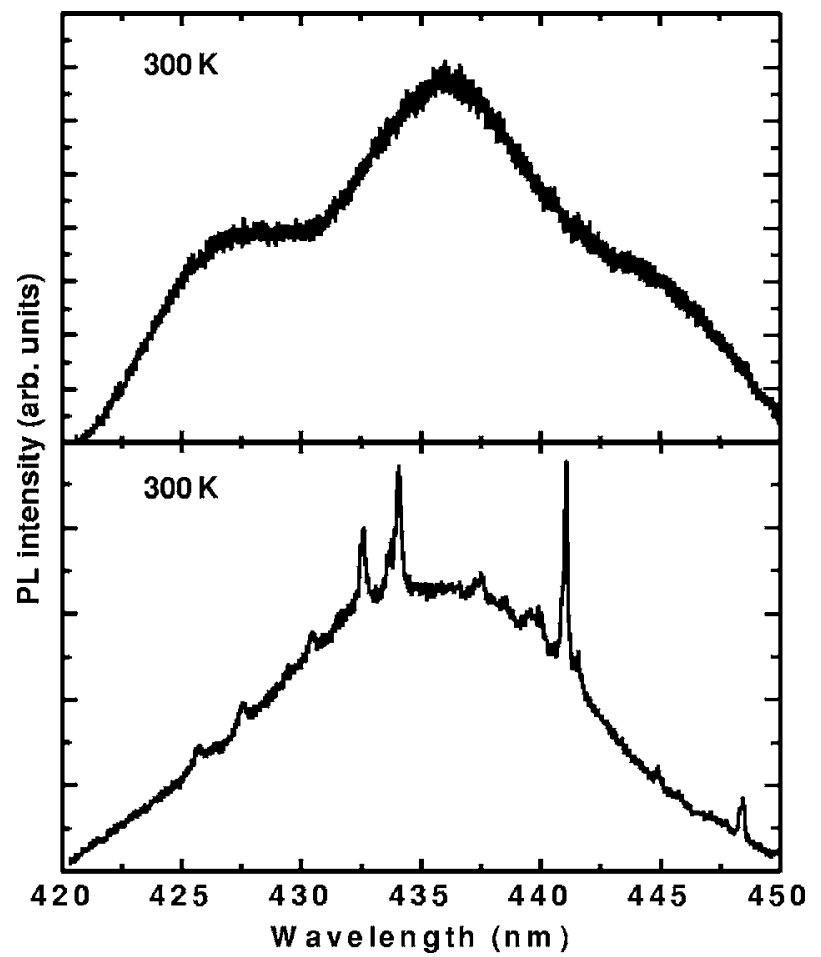

FIG. 2. (a) RT photoluminescence spectrum of bare InGaN/GaN QWs. (b) Multimode structure of a single $4 \mu \mathrm{m} \mu$ disk. The typical mode linewidth is $\sim 0.1 \mathrm{~nm}$ which corresponds to a quality factor $\sim 4000$.

are promising for both CQED studies and the achievement of low-threshold nitride-based lasers.

It is worth discussing the origin of the parameters limiting the $Q$ factor. The latter can be decomposed as ${ }^{17}$

$$
Q^{-1}=Q_{\mathrm{rad}}^{-1}+Q_{\mathrm{abs}}^{-1}+Q_{\mathrm{scat}}^{-1} \text {. }
$$

The value of $Q_{\text {rad }}$ is directly linked to the ratio of the disk diameter to the mode wavelength. For $\mu$ disks with a diameter of $4 \mu \mathrm{m}$ at $\lambda \sim 440 \mathrm{~nm}$, this value exceeds $10^{25}$ and consequently does not limit the performance of our system., ${ }^{2,17}$ The value of $Q_{\text {abs }}$ is intrinsic to the grown materials and is defined as $Q_{\mathrm{abs}}^{-1}=\alpha \lambda / 2 \pi n_{\mathrm{eff}}$, where $\alpha$ is the mean absorption coefficient in the cavity. Absorption usually occurs both in the guiding layer and in the QWs. If QW absorption was the dominant factor, we should observe a narrowing of the optical modes when increasing the pumping power until QWs reach the transparency regime. ${ }^{16}$ Since we do not observe such a behavior for over four decades of excitation power change we can conclude that the impact of QW absorption on $\alpha$ is marginal for these narrow modes. This is in agreement with the large Stokes shift usually reported for InGaN QWs arising mainly from the quantum confined Stark effect. ${ }^{18}$ Furthermore, it reminds the weak modal absorption observed in microdisks using QDs as an active region, ${ }^{16}$ a picture consistent with the QD-like behavior seen in InGaN/GaN QWs. ${ }^{19}$ Consequently in this case the average absorption coefficient in the cavity does match closely that of GaN. For the studied wavelengths, the typical value for the $\mathrm{GaN}$ sub-band-gap absorption ranges between 20 and $40 \mathrm{~cm}^{-1},{ }^{20}$ leading to values for $Q_{\text {abs }}$ comprising between 16000 and 8000, respectively. However, a stronger absorption is expected in the QWs at higher energy due to the reduced Stokes shift between their absorption and emission. This could explain why high quality factors are only 

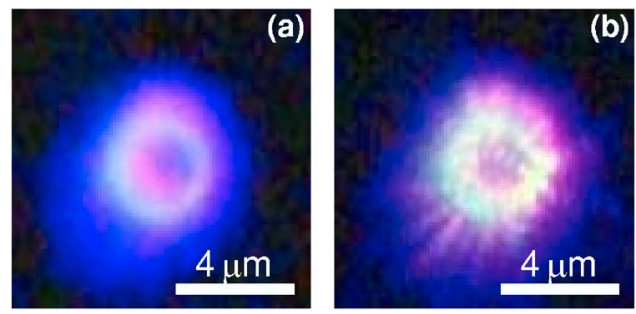

FIG. 3. (Color online) Optical microscope images of a $4 \mu \mathrm{m} \mu$ disk under homogeneous (Gaussian beam) excitation: (a) below and (b) above lasing threshold.

measured on the low energy part of the PL spectra [Fig. 2(b)]. For the low energy modes we can estimate $Q_{\text {scat }}$, which represents the combined influence of surface scattering, external coupling (template) and light extraction. Using Eq. (1), the obtained value is in the range of 4000-8000. This means that the influence of absorption and that of leaky modes are comparable and cannot be easily discriminated. The values found for $Q_{\text {scat }}$ can, however, be tentatively ascribed to an increased light extraction in the vertical direction due to the inclined sidewall. Indeed, under homogeneous laser excitation an increased extraction of the luminescence near the disk edge is observed in optical microscope images [Fig. 3(a)]. The width of this bright ring, about half a micron, corresponds to that of the in plane projection of the sidewall [Fig. 1(b)].

Lasing action was investigated under pulsed excitation. The power dependence of the integrated intensity of the lasing mode as well as its line shape are presented in Figs. 4(a) and 4(b), respectively. It should be noted that laser action has been only achieved for modes in the high energy part of the QW PL spectra which is consistent with the larger absorption in this spectral region, i.e., a larger oscillator strength. A superlinear power dependence, corresponding to single mode lasing operation at $422.5 \mathrm{~nm}$, is observed for an average power density $P_{\mathrm{av}}>1.5 \mathrm{~W} / \mathrm{cm}^{2}$ at RT. The threshold is found at a peak power density of $400 \mathrm{~kW} / \mathrm{cm}^{2}$. For densities superior to $2 \mathrm{MW} / \mathrm{cm}^{2}$, a degradation and even a complete collapse of the lasing mode are observed. Such behavior is attributed to overheating of the $\mu$ disk samples causing their mechanical failure. However a stable lasing action at room
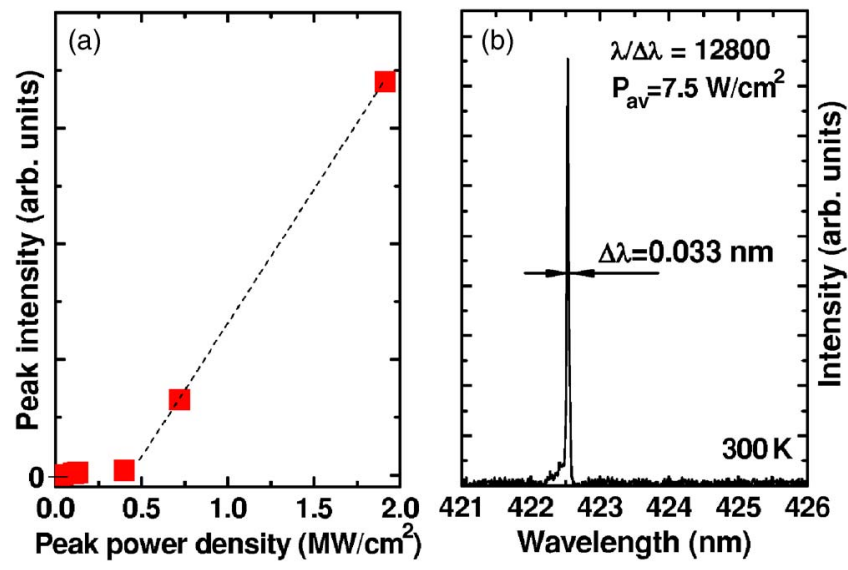

FIG. 4. (Color online) (a) Mode intensity plotted as a function of the peak power density demonstrating a laser threshold at $400 \mathrm{~kW} / \mathrm{cm}^{2}$. (b) $\mu$ disk emission line above threshold. temperature is observed for a range of powers up to four to five times the threshold power which is a significant improvement over what has been previously reported for nitride $\mu$ disks. ${ }^{13}$ This improvement is assigned to increased heat sink capabilities of the present $\mu$ disks due to their large pedestal. The measured linewidth of the laser mode under strong excitation $\left(\sim 1.8 \mathrm{MW} / \mathrm{cm}^{2}\right.$ peak power density) in the stable lasing regime goes down to $0.033 \mathrm{~nm}$, i.e., $\lambda / \Delta \lambda \sim 12800$ [Fig. 4(b)]. Finally the light emission pattern above lasing threshold in the normal direction is presented in Fig. 3(b). The structured pattern demonstrates well coherent light emission.

In conclusion, we have demonstrated the fabrication of III-V nitride $\mu$ disk resonators in the blue wavelength range. Narrow WGMs with a typical quality factor of $\sim 4000$ are observed. RT lasing action was achieved under pulsed optical excitation for a peak power density at threshold as low as $400 \mathrm{~kW} / \mathrm{cm}^{2}$. In the stable lasing regime, the linewidth is less than $0.033 \mathrm{~nm}$. Such system seems to be promising with regard to its light extraction and thermal properties.

The authors are grateful to M. Ilegems for his continuous support. This work was carried out in the framework of the NCCR Quantum Photonics program and the Swiss National Science Foundation. Two of the authors (N.G. and R.B.) are indebted to the Sandoz Family Foundation for its support.

${ }^{1}$ J. L. Jewell, A. Scherer, S. L. McCall, Y. H. Lee, S. Walker, J. P. Harbison, and L. T. Florez, Electron. Lett. 25, 1123 (1989).

${ }^{2}$ S. L. McCall, A. F. J. Levi, R. E. Slusher, S. J. Pearton, and R. A. Logan, Appl. Phys. Lett. 60, 289 (1992).

${ }^{3}$ J. P. Reithmaier, G. Sek, A. Löffler, C. Hofmann, S. Kuhn, S. Reitzenstein, L. V. Keldysh, V. D. Kulakovskii, T. L. Reinecke, and A. Forchel, Nature (London) 432, 197 (2004).

${ }^{4}$ E. Peter, P. Senellart, D. Martrou, A. Lemaître, J. Hours, J.-M. Gérard, and J. Bloch, Phys. Rev. Lett. 95, 067401 (2005).

${ }^{5}$ J.-M. Gérard, B. Sermage, B. Gayral, B. Legrand, E. Costard, and V. Thierry-Mieg, Phys. Rev. Lett. 81, 1110 (1998).

${ }^{6}$ B. Gayral, J.-M. Gérard, B. Sermage, A. Lemaître, and C. Dupuis, Appl. Phys. Lett. 78, 2828 (2001).

${ }^{7}$ M. Pelton, C. Santori, J. Vučković, B. Zhang, G. S. Solomon, J. Plant, and Y. Yamamoto, Phys. Rev. Lett. 89, 233602 (2002).

${ }^{8}$ A. Kavokin and B. Gil, Appl. Phys. Lett. 72, 2880 (1998).

${ }^{9}$ G. Christmann, D. Simeonov, R. Butté, E. Feltin, J.-F. Carlin, and N. Grandjean, Appl. Phys. Lett. 89, 261101 (2006).

${ }^{10}$ T. Someya, R. Werner, A. Forchel, M. Catalano, R. Cingolani, and Y. Arakawa, Science 285, 1905 (1999).

${ }^{11}$ R. Butté, E. Feltin, J. Dorsaz, G. Christmann, J.-F. Carlin, N. Grandjean, and M. Ilegems, Jpn. J. Appl. Phys., Part 1 44, 7207 (2005).

${ }^{12}$ R. A. Mair, K. C. Zeng, J. Y. Lin, H. X. Jiang, B. Zhang, L. Dai, A. Botchkarev, W. Kim, H. Morkoç, and M. A. Khan, Appl. Phys. Lett. 72, 1530 (1998).

${ }^{13}$ E. D. Haberer, R. Sharma, C. Meier, A. R. Stonas, S. Nakamura, S. P. DenBaars, and E. L. Hu, Appl. Phys. Lett. 85, 5179 (2004).

${ }^{14}$ J. Dorsaz, H.-J. Bühlmann, J.-F. Carlin, N. Grandjean, and M. Ilegems, Appl. Phys. Lett. 87, 072102 (2005).

${ }^{15}$ J.-F. Carlin and M. Ilegems, Appl. Phys. Lett. 83, 668 (2003).

${ }^{16}$ B. Gayral, J.-M. Gérard, A. Lemaître, C. Dupuis, L. Manin, and J. L. Pelouard, Appl. Phys. Lett. 75, 1908 (1999).

${ }^{17}$ R. E. Slusher, A. F. J. Levi, U. Mohideen, S. L. McCall, S. J. Pearton, and R. A. Logan, Appl. Phys. Lett. 63, 1310 (1993).

${ }^{18}$ E. Berkowicz, D. Gershoni, G. Bahir, E. Lakin, D. Shilo, E. Zolotoyabko, A. C. Abare, S. P. Denbaars, and L. A. Coldren, Phys. Rev. B 61, 10994 (2000).

${ }^{19}$ H. Schömig, S. Halm, A. Forchel, G. Bacher, J. Off, and F. Scholz, Phys. Rev. Lett. 92, 106802 (2004).

${ }^{20}$ F. Omnès, N. Marenco, B. Beaumont, Ph. de Mierry, E. Monroy, F. Calle, and E. Muñoz, J. Appl. Phys. 86, 5286 (1999). 